\title{
In Situ TEM Measurements of the Mechanical Properties and Behavior of $\mathrm{WS}_{2}$ Nanotubes
}

\author{
Ming Sheng Wang ${ }^{1}$, Ifat Kaplan-Ashiri ${ }^{2}$, Xian Long Wei ${ }^{1}$, Rita Rosentsveig ${ }^{2}$, Hanoch Daniel Wagner ${ }^{2}$, Reshef \\ Tenne $^{2}(\square)$, and Lian Mao Peng ${ }^{1}$ \\ ${ }^{1}$ Key Laboratory on the Physics and Chemistry of Nanodevices and Department of Electronics, Peking University, Beijing 100871, China \\ ${ }^{2}$ Department of Materials and Interfaces, Weizmann Institute of Science, Rehovot 76100, Israel \\ Received: 28 April 2008/ Revised: 25 May 2008/Accepted: 25 May 2008 \\ CTsinghua Press and Springer-Verlag 2008
}

\begin{abstract}
The mechanical properties of individual $\mathrm{WS}_{2}$ nanotubes were investigated and directly related to their atomic structure details by in situ transmission electron microscope measurements. A brittle mode deformation was observed in bending tests of short (ca. $1 \mu \mathrm{m}$ in length) multilayer nanotubes. This mode can be related to the atomic structure of their shells. In addition, longer nanotubes (6-7 $\mu \mathrm{m}$ in length) were deformed in situ scanning electron microscope, but no plastic deformation was detected. A "sword-in-sheath" fracture mechanism was revealed in tensile loading of a nanotube, and the sliding of inner shells inside the outermost shell was imaged "on-line". Furthermore, bending modulus of 217 GPa was obtained from measurements of the electric-fieldinduced resonance of these nanotubes.
\end{abstract}

\section{KEYWORDS}

Inorganic nanotubes, nanomechanics, TEM, SEM

\section{Introduction}

In spite of the great technological progress that was made during the last years, comprehensive characterization of nanomaterials is still a challenging task, let alone the coupling between different properties (e.g., electromechanical properties). Imaging tools like scanning electron microscopy (SEM) and scanning force microscopy (SFM) can also be utilized as measurement tools. Hence, they play a central role in physical properties characterization of individual nanoparticles. Moreover, a combination of two different imaging techniques, like scanning tunneling microscopy (STM) and transmission electron microscopy (TEM) provide us with a very powerful tool for studying the coupling between the material's physical properties and its structural habits.

The mechanical properties of individual nanostructures can be thus studied directly by using variety of microscopy techniques, each one providing a different aspect of their characteristics. For instance, the first mechanical test of individual carbon nanotubes was performed by in situ experiments in the TEM. Treacy et al. [1] estimated the nanotubes' Young's modulus by measuring the amplitude of their intrinsic thermal vibrations. Furthermore, TEM studies produced direct observation of various deformation and fracture modes under compression of single multiwalled carbon nanotubes [2]. Pulling and bending tests on individual carbon nanotubes

Address correspondence to reshef.tenne@weizmann.ac.il 
by in situ TEM measurements were conducted. Here a special tensile testing stage was developed for this purpose, using microfabrication techniques [3]. This device enabled the direct application of a tensile strain to individual nanotubes while they are viewed with close to an atomic resolution by the TEM. In a very advanced study, starting from an amorphous carbon film, single-walled carbon nanotubes were obtained in situ in a high resolution transmission electron microscopy (HRTEM) by the combined effect of irradiation and axial strain [4]. These nanotubes were pulled and their mechanical behavior was characterized. A recent study was devoted to the kink formation and motion in carbon nanotubes at high temperatures and was carried out inside an HRTEM [5, 6].

The first inorganic nanotubes and fullerenelike nanoparticles, made of $\mathrm{WS}_{2}$ were discovered in 1992 [7]. Elucidating their growth mechanism from tungsten oxide nanoparticles permitted the scalingup of their production, which led to their recent commercialization as superior solid lubricants [8]. This progress makes the study of their mechanical properties highly warranted. Indeed, the mechanical properties and behavior of $\mathrm{WS}_{2}$ nanotubes were studied experimentally as well as by simulations and $\mathrm{ab}$ initio calculations. The Young's modulus of $\mathrm{WS}_{2}$ nanotubes was measured using different techniques. Thus, buckling tests resulted in an average value of $171 \mathrm{GPa}$ [9] and tensile and post buckling tests resulted in a value of $150 \mathrm{GPa}$ [10]. These Young's moduli values are in good agreement with density functional-tight binding (DFTB) calculations as well. The Young's modulus reflects the strength of the chemical bonds, both in nano and macro scale. Furthermore, the nanotubes exhibited high tensile strength (16 GPa); large values of strain (14\%), as well as large elastic deformations. This behavior is unique to the defect-free nanotubes and do not characterize the bulk material, which is full with defects of different kinds [10]. Moreover, the sliding modulus between different walls of the $\mathrm{WS}_{2}$ nanotubes (which was determined by bending tests), was found to be smaller (2 GPa) compared to that of bulk $\mathrm{MoS}_{2}$ (19 GPa) [11]. It is believed that the (telescopic) sliding of the defectfree multiwall nanotubes is dictated by pure van der
Walls interaction between the shells. Contrarily, the bulk material contains substantial amounts of defects, which pin the layers together, preventing thereby their almost free sliding [11].

Shock wave resistance measurements of $\mathrm{WS}_{2}$ nanotubes were carried out as well [12]. It was found that $\mathrm{WS}_{2}$ nanotubes are capable of withstanding stress caused by shock waves of up to 21-25 GPa. X-ray diffraction (XRD) and Raman analyses have confirmed that unlike carbon nanotubes, which were shown to decompose and transform into a diamond phase under appreciably smaller stress condition (9 GPa), the structural integrity of the $\mathrm{WS}_{2}$ nanotubes under shock is preserved, except for some delimitation of the outermost layers.

So far, the mechanical tests of $\mathrm{WS}_{2}$ nanotubes were performed within the atomic force microscopy (AFM) and the SEM. Due to the resolution limitation of the SEM, the nanotubes' structural data before and during the buckling, bending and tensile tests could not be resolved clearly. In the present study, the mechanical behavior of multiwalled $\mathrm{WS}_{2}$ nanotubes was studied by in situ TEM experiments. The deformation process and fracture in some mixed modes of bending and buckling were revealed as well as the "sword-insheath" failure mechanism in tensile tests. These mechanical characteristics further confirm the unique properties of the nanotubular phase of $\mathrm{WS}_{2}$.

\section{Experimental}

In situ TEM mechanical tests were carried out in a 200-kV TEM (Tecnai G20) with a single-tilt sample holder (Nanofactory) integrated with a scanning tunneling microscope. In a typical experiment, two metal electrodes were used. The first electrode was a microscopically flat Pt tip that was prepared by simply cutting a pure Pt wire of $0.3 \mathrm{~mm}$ diameter using a scissor. The second electrode was a very sharp W-tip with a radius of curvature of several tens nanometers, which was prepared by using the standard electrochemical etching method. The W-tip was connected to a piezotube that allows a fine movement in three dimensions over several micrometers. The $\mathrm{WS}_{2}$ nanotubes were assembled onto the Pt tip before each experiment by rubbing the Pt-tip in the 
nanotube powder. The microscopically flat Pt-tip was kept stationary while individual $\mathrm{WS}_{2}$ nanotubes were selected by the sharp W-tip inside the TEM. The vacuum in the TEM was $1 \times 10^{-5} \mathrm{~Pa}$. Amorphous carbon $(\mathrm{a}-\mathrm{C})$ was deposited $[13,14]$ (for example, to fix the nanotubes on the electrodes) by focusing the electron beam on the area of interest with a small spot size.

In situ TEM bending tests of $\mathrm{WS}_{2}$ nanotubes, by the electric-field-induced resonance method were performed, as described before for carbon nanotubes [15-19]. A single selected $\mathrm{WS}_{2}$ nanotube was fixed on a W-tip by deposition of a-C. The distance between the nanotube and the top $\mathrm{Pt}$ wire was about several micrometers or even closer, and the nanotube was not perpendicular to the counter electrode. A tunable sinusoidal signal $\left(V_{\mathrm{ac}} \leqslant 10 \mathrm{~V}\right)$ provided by the function generator was applied across the nanotube and the counter electrode. By adjusting the applied frequency, the nanotube was brought to a resonance with the field.

In situ SEM experiments were carried out as well in the LEO Supra 55 high resolution SEM (HRSEM) equipped with a nanomanipulator (Klocke Nanotechnik; $10 \mathrm{~nm}$ steps). First, a $\mathrm{WS}_{2}$ nanotube was attached to a silicon cantilever within the environmental SEM (E-SEM model FEI XL-30 equipped with a micro-manipulation system-Model Quarter Research \& Development). The nanotubes were attached to Si cantilevers by exposing the contact point to the electron beam, producing amorphous carbon from hydrocarbons in the vacuum to form high strength glue [9, 10]. Later on the nanotube tip was moved to the HRSEM, there a mirror polished silicon wafer was pushed against it. The nanotube was pictured following each movement [10].

\section{Results and discussions}

\subsection{Bending and fracture of $\mathrm{WS}_{2}$ nanotubes}

In situ TEM bending and buckling studies of individual $\mathrm{WS}_{2}$ nanotubes were performed. Qualitative mechanical characterization of individual $\mathrm{WS}_{2}$ nanotubes was carried out by means of deformation applied on a fixed nanotube. The nanotubes were deformed along arbitrary paths, usually in some sort of bending. Since the system is not equipped with a force sensor, the loads which were applied on the nanotubes are unknown. Still the results presented in Figs. 1 and 2 give a new insight into the mechanism of deformation and failure of these nanotubes.

A deformation process of a $\mathrm{WS}_{2}$ nanotube within the TEM is presented in Fig. 1. This nanotube is ca. $1.5 \mu \mathrm{m}$ long and $21 \mathrm{~nm}$ in diameter. The nanotube was attached to the tungsten probe (amorphous carbon served as glue) at one end. The other end was just touching the Pt electrode, hence it was held only by surface forces. Therefore, a fixed-free configuration can be determined for this experimental system. A bent nanotube is presented in Fig. 1(a), its profile resembles slender column in the second mode of

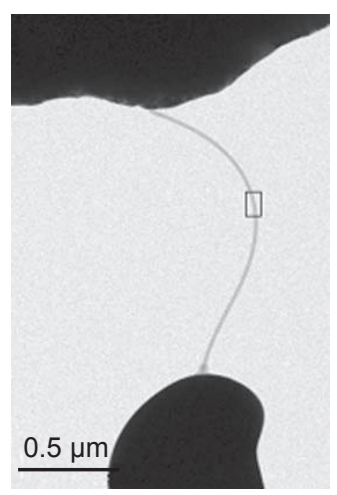

(a)

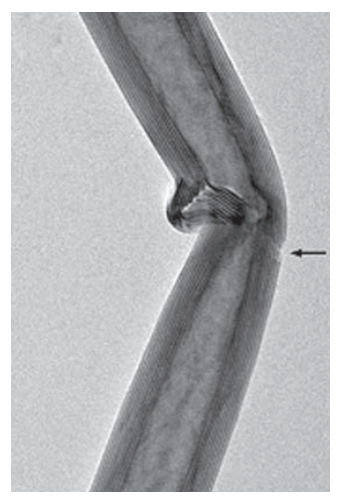

(d)

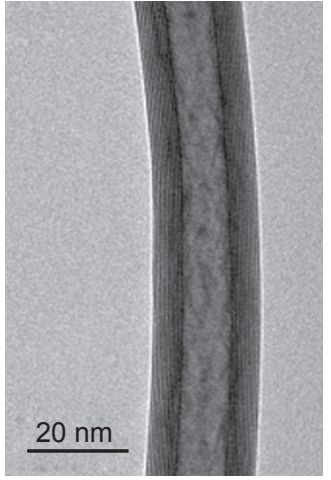

(b)

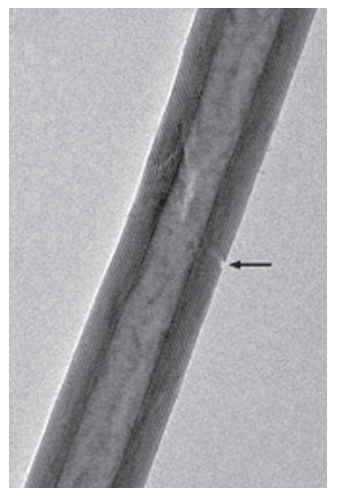

(e)

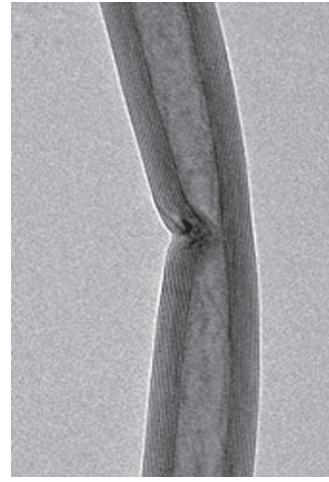

(c)

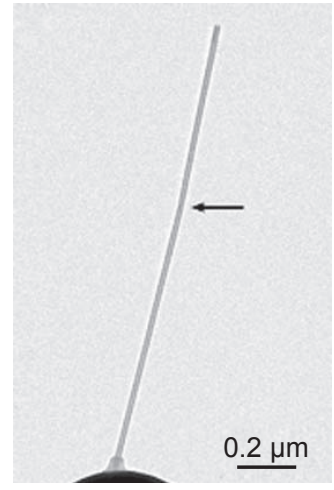

(f)

Figure 1 (a) A bent $W_{2}$ nanotube with one end fixed on W-tip with amorphous c. (b)-(e) A series of HRTEM images recording the deformation process of the nanotube segment marked in (a). The arrows indicate the rip in the outmost wall 
buckling [20]. The load for this mode of buckling can be estimated according to the equation [20]:

$$
P_{\text {cr }}=\frac{9 \pi^{2} E I}{4 L^{2}}
$$

where $E$ is the Young's modulus, $I$ is the geometrical moment of inertia $\left(I=\pi / 4\left(R_{\text {out }}^{4}-R_{\text {in }}^{4}\right)\right.$ where $R_{\text {out }}$ and $R_{\text {in }}$ are the nanotube's outer and inner radius, respectively), and $L$ is the nanotube's length. Substituting the nanotube's dimensions and its Young's modulus [9, 10] (150 GPa) a critical load of $10 \mathrm{nN}$ is observed. Hence, the applied loads can be evaluated according to the nanotube's profile.

A higher magnification of the marked area is presented in Fig. 1(b). It can be seen that the nanotube buckled uniformly, with a local radius of curvature about $320 \mathrm{~nm}$. The local strain of the nanotube can be estimated according to the equation:

$$
\varepsilon={ }^{R_{\text {out }}} / R_{\mathrm{c}}
$$

where $R_{\text {out }}$ is the radius of the tube and $R_{\mathrm{c}}$ is its radius of curvature during bending. According to Fig. 2(b), the local strain $\varepsilon$ on the outside surface of the bent $\mathrm{WS}_{2}$ nanotube $\left(R_{\text {out }}=10.5 \mathrm{~nm}, R_{\mathrm{c}}=320 \mathrm{~nm}\right)$ is estimated to be $3 \%$. Under further deformation, the nanotube became unstable and collapsed to form a single kink (Fig. 1(c)). The local strain can be estimated again according to Eq. (2). Now $R_{\mathrm{c}} \approx 150 \mathrm{~nm}$ and the strain can be evaluated as $7 \%$. Upon further increasing the load (Fig. 1(d)), the outermost layers could not sustain the tensile strain, and a rip occurred (indicated by an arrow). As larger load was applied on the nanotube, the consequent rupture of the layers on the stretched side was observed until the point of local failure. At this point the strain of the nanotube can be estimated as $13 \%-14 \%$. Next, the load was released by retracting the $\mathrm{W}$-tip and a rip occurred at the outermost layers. As the nanotube was detached from the Pt wire it was recovered to its original straight shape, except for the rip in the outermost layer. According to previous measurements strain values in the range of $8 \%-14 \%$ were observed at the failure point [10], in tensile tests of $\mathrm{WS}_{2}$ nanotubes. Since no plastic deformation or failure can be observed in Figs. 1(a)-(b), the estimated strain is compatible with the previous data $[10,11]$. Furthermore, the higher strain value that was evaluated for the nanotube when it was further deformed (Fig. 1(c)) ca. 7\%, almost approaches the lower limit value for fracture of $\mathrm{WS}_{2}$ nanotubes. At higher loads only the outermost layers, which suffered the highest strain (as illustrated in Fig. 1(d)), completely failed. No defects were observed at the inner shells of the nanotubes; hence it was able to recover to its straight shape. The high fracture resistance of the multiwalled tubular structure is clearly revealed here.

The fracture evolution in another $\mathrm{WS}_{2}$ nanotube, ca. $1 \mu \mathrm{m}$ long, is demonstrated using a series of TEM pictures in Fig. 2. The undeformed nanotube and its magnified ends are presented in Figs. 2(a)-(c). The amorphous carbon which serves as a glue $[13,14]$ between the nanotube and the two contacts (Figs. 2(b)-(c)) is clearly observed. By moving the tungsten probe towards the Pt electrode, a load was applied on the nanotube, and two centers of deformation in the form of kinks appeared (Fig. 2(d)). Upon further increasing the load the layers broke apart in both centers of deformation (Figs. 2(e), (g), (h)). It is clearly demonstrated that the failure was stimulated on the stretched part (as was also observed in Fig. 1). Other segments of the nanotubes were completely undeformed and maintain their linear structure. A catastrophic failure then occurred on the lower kink site (Fig. 2(f)), and resulted in two shorter parts of the original nanotube. The upper part of the nanotube sustained the deformed structure.

Different mechanical behaviors were observed for the tested nanotubes. The first nanotube was able to buckle in the second mode without any visible structural changes. Furthermore, upon further loading, only partial failure occurred and the nanotube maintained its straight shape as the load was released. The second nanotube, which was shorter (by ca. 30\%) was plastically deformed and completely failed under bending. Still, in both nanotubes kinks were formed. The differences can be attributed to the dissimilar nanotubes' length (rigidity) and the loading conditions.

Former studies of $\mathrm{WS}_{2}$ nanotubes both under postbuckling [10] and bending [11] showed a nonlinear elastic behavior. Presumably, this nonlinearity can be attributed to the kinks that tend to form in the tubular structure which was confirmed by the in situ

\section{Springer}




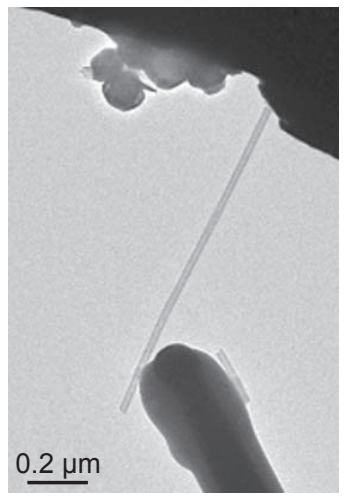

(a)

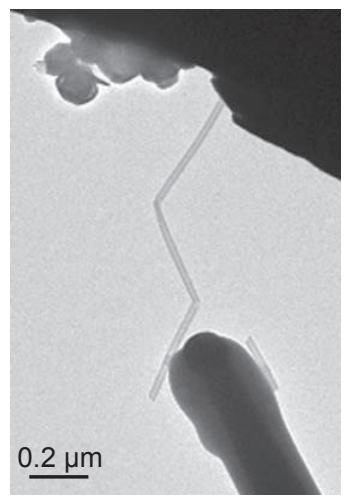

(d)

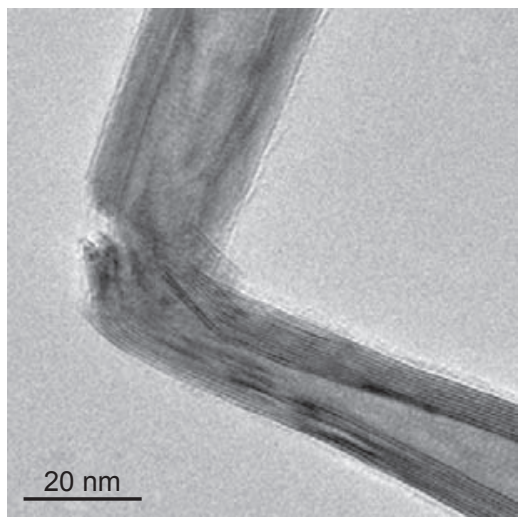

(g)

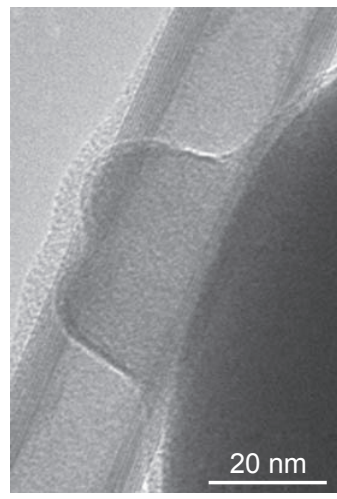

(b)

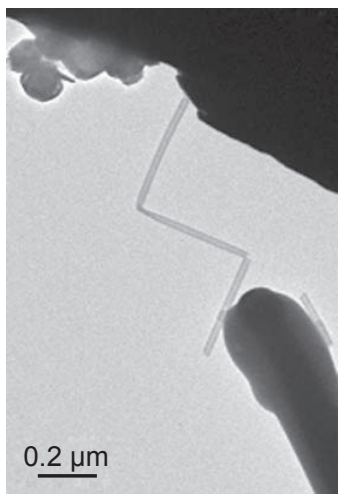

(e)

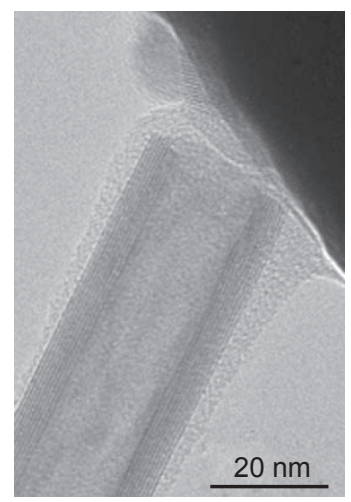

(c)

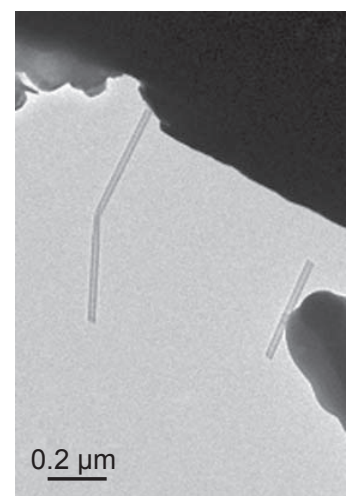

(f)

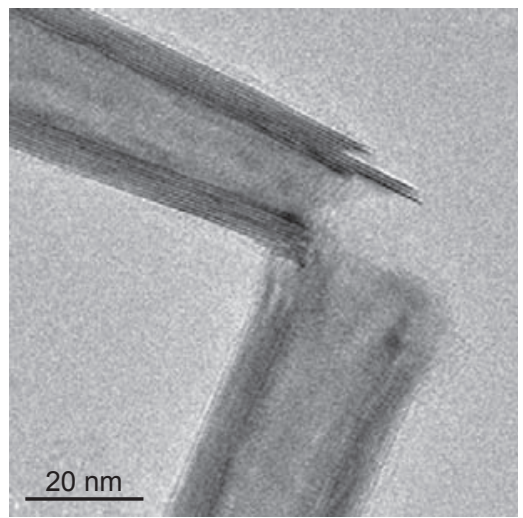

(h)
Figure 2 TEM image of an individual $W_{2}$ nanotube (a) with its two ends fixed on W- tip (b) and Pt wire (c), respectively. The nanotube was compressed and two kinks were formed (d), (e) finally it was fractured (f). (g) and (h) are HRTEM images of the two kinks in (e)

TEM experiments (Figs. 1 and 2).

In situ SEM buckling studies of individual $\mathrm{WS}_{2}$ nanotubes were performed as well. A load was applied on the nanotube by pushing a silicon surface against it. The experiment was performed several times on different nanotubes; a representative sample is presented in Fig. 3. First the nanotube $(6-7 \mu \mathrm{m}$ in length) was deflected in the plane perpendicular to the image plane (Fig. 3(a)). As the nanotube was further loaded it became highly deformed resulting in the formation of a loop (Fig. 3(b)). The unloading process of the nanotube is presented in Figs. 3(c) and (d). As the nanotube was completely unloaded it jumped immediately to the tip, forming again a loop shape (not shown here). No fracture occurred during this deformation process. The limited resolution of the SEM does not allow determining either or not a plastic deformation occurred in this experiment.

The great flexibility of the $\left(\mathrm{WS}_{2}\right)$ nanotubular morphology is clearly demonstrated here. In contrary to the nanotubes, the bulk material is very brittle and would suffer only small deformations before fracture. Furthermore, the large nanotube's deformation, which was observed only at in situ SEM experiments, can be related to the nanotube's length. Other factors like the radius and the density of defects are non-relevant here since in all the experiments the nanotubes have similar radii (ca. $20 \mathrm{~nm}$ ) and are considered as defect free [10].

A similar behavior was observed for carbon nanotubes which was denoted by large amplitude deformations beyond the Hookean behavior [21]. The nanotubes developed kinks or ripples (multiwalled tubes) under compression and bending strain. They furthermore flatten into deflated ribbons under torsion, and still could reversibly restore their original shape upon releasing the stress $[2,3,16,21-25]$. This behavior is attributed to the remarkable flexibility of the hexagonal network, which resists bond breaking and bond switching up to very high strain values [24]. Furthermore, the small dimensions of the nanotubes, leaves no room for the common macroscopic failure modes, which involves stress-concentrators like microcracks or dislocation 


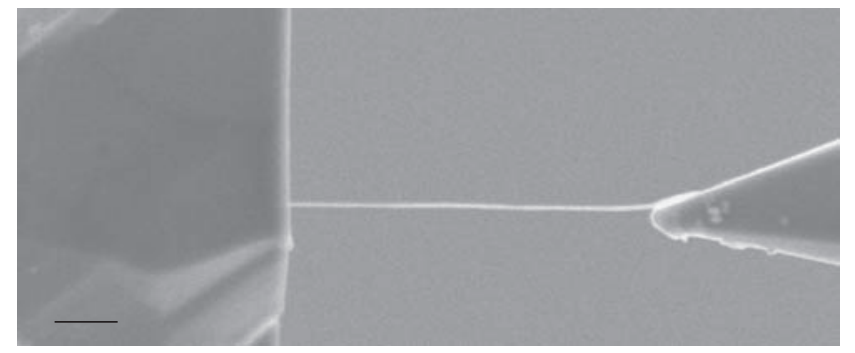

(a)

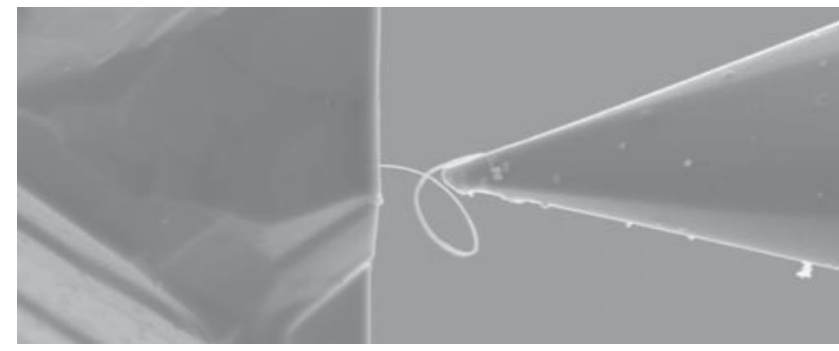

(b)

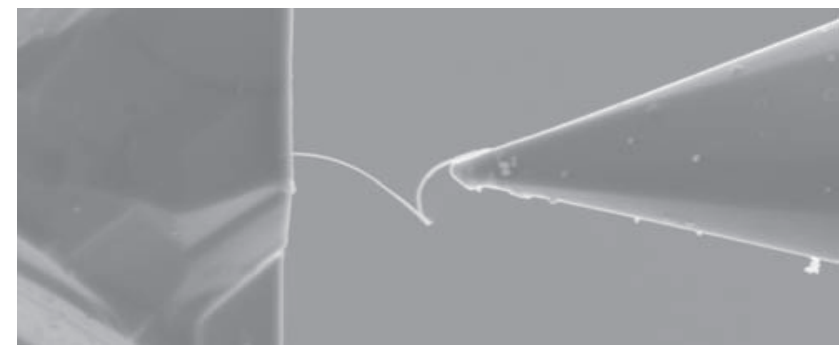

(c)

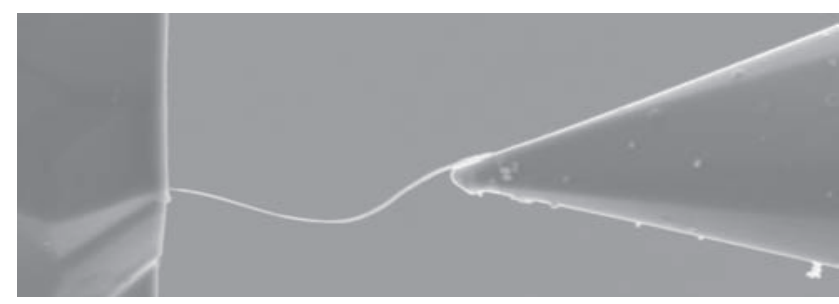

(d)

Figure 3 A series of HRSEM images recording the deformation process of an individual $W_{2}$ nanotube: (a) and (b) present the loading process while (c) and (d) present the unloading process. Scale bar is $1 \mu \mathrm{m}$

failure piles [21]. A variety of experimental evidence confirms that nanotubes can sustain significant nonlinear elastic deformations [2, 3, 16, 21-25]. Lourie et al. [2] showed that under large bending, carbon nanotubes collapse to form kinks on the internal (compressive strain) side of the bent nanotube, in full agreement with theoretical predictions. Moreover, long and slender multiwalled carbon nanotubes which are under compression strain, behave as elastica rods and form bends and loops [2]. Kink formation was also observed for BN nanotubes [26]. In situ TEM bending of $\mathrm{BN}$ nanotubes to angles beyond $30^{\circ}-40^{\circ}$ resulted in elastic deformation which proceeded through the propagation of consecutive momentary kinks.

Similar behavior was observed for $\mathrm{WS}_{2}$ nanotubes. A ripple mode did not occur, which might be the result of the $\mathrm{WS}_{2}$ layer's stiffness. The high stiffness value can be attributed to the sandwich $\mathrm{S}-\mathrm{W}-\mathrm{S}$ atomic structure of the nanotube layer.

An attempt to verify the mode of fracture of the $\mathrm{WS}_{2}$ nanotube as revealed from Figs. $2(\mathrm{~g})-(\mathrm{h})$ is made. It is impossible to locate in these images dislocations or any other sign of plastic deformation. Some of the inner layers seem to slide out. Former studies on these nanotubes, showed a brittle mode of fracture in tensile tests [10]. Also, molecular dynamics (MD) simulation of singlewalled $\mathrm{MoS}_{2}$ showed that the fracture occurred on its circumference without any plastic deformation [10]. Hence, the fracture in this case can be considered as a brittle one. In a similar study on carbon nanotubes, bending of both arc discharge made multiwalled carbon nanotubes (MWCNTs, Arc-CNT) as well as chemical vapor deposition (CVD) made MWCNTs (CVD-CNT) was conducted by in situ TEM. The Arc-CNT showed local plastic deformation over the elastic limit, while that of the CVD-CNT were failed in a brittle fracture mode. The difference in their mechanical characteristics was attributed to the atomic structure of the defects on the cylindrical wall of the two kinds of nanotubes and to their stiffness according to the difference in a diameter (number of the graphitic walls) [27]. A recent study also showed yielding in tensile test of Arc-CNT [28]. In an earlier study it was suggested that a nanotube deforms elastically until certain critical curvature is attained; then the atomic bonding in the stressed side changes from a graphite-like bonding state to a diamond-like state [29] (in accordance with the phase diagram of carbon which demonstrate phase transformation of graphite to diamond at a pressure of $1.7 \mathrm{GPa}$ at room temperature [30]). Since, no high pressure phase exists for $\mathrm{WS}_{2}$, this kind of plastic deformation is not likely to occur in this nanotubes making them very stable under high pressure [31]. 


\subsection{Tensile loading of $\mathrm{WS}_{2}$ nanotubes}

Tensile test of individual $\mathrm{WS}_{2}$ nanotube, by in situ TEM experiments, is described in this part. The nanotube's ends were fixed to the electrodes with a-C as described before. By moving the tungsten probe, a load was applied and consequently the nanotube was strained and elongated. As the load was further increased the nanotube failed and finally fractured.

Figure 4 presents a set of TEM images, which describe the process of pulling an individual $\mathrm{WS}_{2}$ nanotube. Before the test, the nanotube has a uniform diameter consisting of 9 layers (Fig. 4(a)). Figures 4(b)(e) demonstrate clearly that a failure occurred on the nanotube's outer shell. This failure mechanism is the so-called "sword-in-sheath" fracture mechanism $[32,33]$. Following the fracture of the nanotube, a sliding event occurs between the inner shells and the outmost layer in a telescopic fashion (Fig. 4(b)). HRTEM image in Fig. 4(c) demonstrates clearly that the "sword" is now still inside the "sheath". Finally, the internal shells have been totally pulled away from the top fragment, leaving a single-walled nanotube (Fig. 4(d)). The arrows in Figs. 4(d)-(e) indicate the fracture site on the outmost layer. As expected, the missing length of the broken shell on the lower multiwalled nanotube is about $200 \mathrm{~nm}$, which is equal to the length of top single-wall nanotube. This sword-in-sheath mode (or telescopic mode) of failure was predicted earlier in tensile tests of $\mathrm{WS}_{2}$ nanotubes, which were carried out in SEM [10]. Unlike the bending deformation which was discussed earlier, in the case of the nanotube's pulling a similar mode of deformation and rupture was observed for both the $\mathrm{WS}_{2}$ and the carbon nanotubes [10, 33, 34].

\subsection{Electric-field-induced resonance of $\mathrm{WS}_{2}$ nanotubes}

In situ TEM bending tests of $\mathrm{WS}_{2}$ nanotubes, by the electric-field-induced resonance method were performed. Here, the fundamental natural frequency of the nanotube is obtained; this frequency

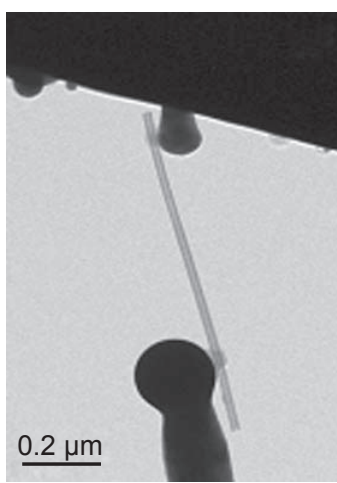

(a)

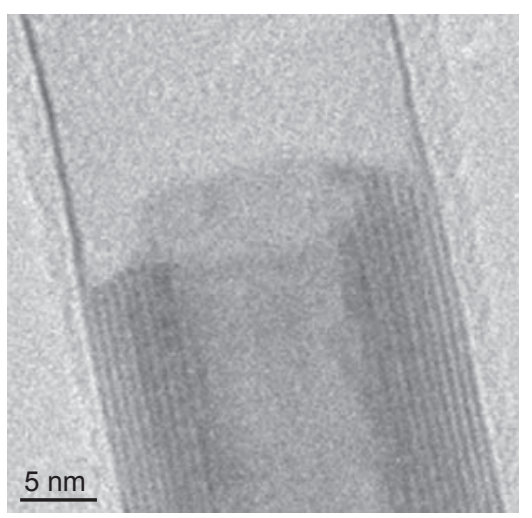

(c)

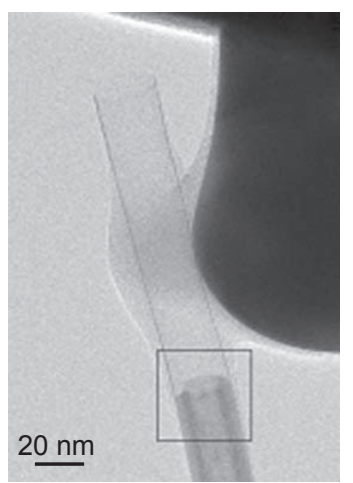

(b)

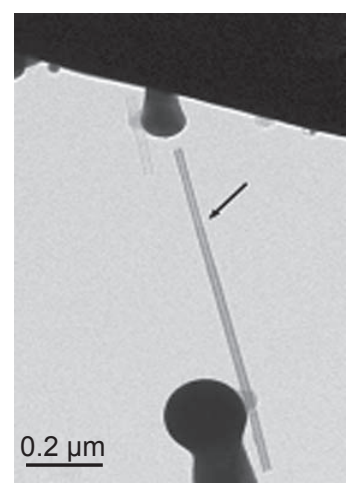

(d)

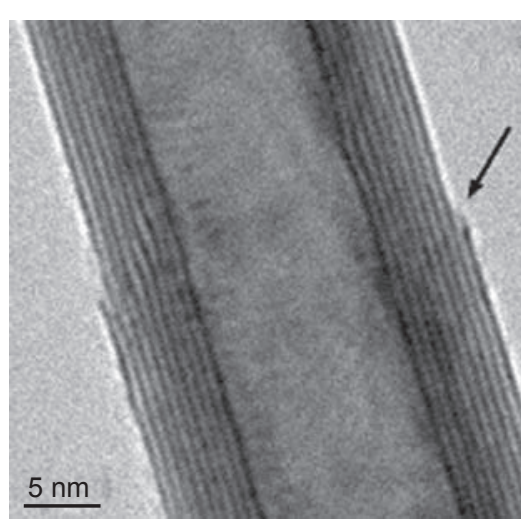

(e)

Figure 4 (a) A single WS with its two ends fixed on two electrodes with amorphous C. After the nanotube was fractured, the inner shells slide inside along (b) and finally pulled out (d) from the outmost layer. (c) and (e) are HRTEM images of the area marked in (b)and (e), respectively is related to the bending modulus of the material by the Euler-Bernoulli equation for a cantilevered beam [16, 35]:

$$
f_{i}=\frac{\beta_{i}^{2}}{8 \pi L^{2}} \sqrt{\frac{\left(D_{\mathrm{I}}^{2}+D_{\mathrm{O}}^{2}\right) E_{\mathrm{b}}}{\rho}}
$$

where $D_{\mathrm{I}}, D_{\mathrm{O}}, L, E_{\mathrm{b}}$, and $\rho$ are the inner and outer diameter, length, bending modulus, and density of the nanotubes, respectively. $\beta_{i}$ is a constant for the $i$ th harmonic, when $\beta_{1}=1.875$ and $\beta_{2}=$ 4.694 [16]. The bulk density of $\mathrm{WS}_{2}$ is $7.73 \mathrm{~g} / \mathrm{cm}^{3}$. Other structural parameters, such as the inner and outer diameter and even the number of the layers, can be accurately measured from the highresolution image of the nanotube's root. All the measured nanotubes were perfectly crystalline and uniform in diameter.

Once, the fundamental natural frequency $f_{1}$ is observed, small vibration amplitude was maintained in order to minimize the bending curvature. Then, the dependence of the amplitude on 
frequency was measured. From these measurements and using Eq. (3) an average value of $(217 \pm 40)$ GPa was observed for the bending modulus, $E_{\mathrm{b}}$. In former studies on CNT's the Young's modulus was considered to be equal to the bending modulus. As for $\mathrm{WS}_{2}$ nanotubes, the value obtained for their bending modulus is slightly higher compared to the Young's moduli determined by using other techniques as well as by DFTB calculations (150$170 \mathrm{GPa})[9,10,36]$. Former bending tests of $\mathrm{WS}_{2}$ nanotubes resulted in a nonlinear elastic behavior for large deflections (as was demonstrated according to the force deflection curves) [11]. The value of the Young's modulus calculated from the maximum deflection of these nanotubes was superfluous. It is concluded therefore, that the bending modulus calculated from large bending deflections is not directly comparable to the Young's modulus of the nanotubes.

Finally, the subject of potential influence of the electron beam on the mechanical loading will be discussed. The high value obtained for the bending modulus of $\mathrm{WS}_{2}$ nanotubes imply that the impact of the electron beam does not damage them. TEM and SEM examination of numerous $\mathrm{WS}_{2}$ nanotubes under up to $300 \mathrm{keV}$ had shown the great stability of their structure under the electron beam. Furthermore, in situ SEM tensile tests of these nanotubes resulted in their theoretical strength, suggesting that electron beam damage does not alter the structure of these nanotubes [10].

\section{Summary}

The mechanical properties of $\mathrm{WS}_{2}$ nanotubes were studied by in situ TEM and SEM experiments. Centers of deformation in the form of kinks occurred while the nanotubes were loaded until complete or partial fracture occurred. These kinks can be correlated to the nonlinear elastic deformations of the nanotubes which were observed before. A hint for the nanotubes' remarkable resistance to fracture was also demonstrated as a rip occurred but it was not propagated. The incredible capability of the tubular morphology to highly deform was demonstrated as well. The deformation mechanism of the $\mathrm{WS}_{2}$ nanotubes is somehow different from the one observed for carbon nanotubes (since no ripples occurred). This difference can be related to the more complex atomic structure of the $\mathrm{WS}_{2}$ layers (the shell width is of 3 atoms) which makes it stiffer than carbon nanotubes.

Tensile test of individual $\mathrm{WS}_{2}$ nanotubes experiments revealed the "sword-in-sheath" or telescopic failure mechanism which was predicted before. According to this mechanism when a pure tension is applied to a multiwalled nanotube which is attached to the tip through its outermost layer only, the failure occurs in this layer only, with the rest of the layers remaining intact.

The bending modulus of $\mathrm{WS}_{2}$ nanotubes was measured by the electric-field-induced resonance method. An average value of $217 \mathrm{GPa}$ was obtained which is slightly higher compared to the Young's modulus (150 GPa) of these nanotubes. A comprehensive analysis of these measurements combined with detailed ab initio calculations will be presented elsewhere.

Further studies of the deformation and fracture of $\mathrm{WS}_{2}$ nanotubes are required in order to fully understand these mechanisms and the effect of defects on their mechanical behavior.

\section{Acknowledgments}

This work was supported by the joint China-Israel (Chinese and Israeli Ministries of Science and Technology) agreement. RT is the holder of the Drake Family Chair in Nanotechnology and the director of the Helen and Martin Kimmel Center of Nanoscale Science. HDW is the incumbent of the Livio Norzi Chair in Materials Science. We also are indebted to the support of the Harold Perlman Foundation and the Irving and Cherna Moskowitz. Center for Nano and Bio-Nano Imaging.

\section{References}

[1] Treacy, M. M. J.; Ebbesen, T. W.; Gibson, J. M. Exceptionally high Young's modulus observed for individual carbon nanotubes. Nature 1996, 381 (6584), 678-680.

\section{基 Springer}


[2] Lourie, O.; Cox, D. M.; Wagner, H. D. Buckling and collapse of embedded carbon nanotubes. Phys. Rev. Lett. 1998, 81 (8), 1638-1641.

[3] Demczyk, B. G.; Wang, Y. M.; Cumings, J.; Hetman, M.; Han, W.; Zettl, A.; Ritchie, R. O. Direct mechanical measurement of the tensile strength and elastic modulus of multiwalled carbon nanotubes. Mat. Sci. Eng. a-Struct. 2002, $334(1-2), 173-178$.

[4] Troiani, H. E.; Miki-Yoshida, M.; Camacho-Bragado, G. A.; Marques, M. A. L.; Rubio, A.; Ascencio, J. A.; JoseYacaman, M. Direct observation of the mechanical properties of single-walled carbon nanotubes and their junctions at the atomic level. Nano Lett. 2003, 3 (6), 751755.

[5] Huang, J. Y.; Chen, S.; Ren, Z. F.; Wang, Z. Q.; Wang, D. Z.; Vaziri, M.; Suo, Z.; Chen, G.; Dresselhaus, M. S. Kink formation and motion in carbon nanotubes at high temperatures. Phys. Rev. Lett. 2006, 97 (7), 075501.

[6] Huang, J. Y.; Chen, S.; Wang, Z. Q.; Kempa, K.; Wang, Y. M.; Jo, S. H.; Chen, G.; Dresselhaus, M. S.; Ren, Z. F. Superplastic carbon nanotubes-Conditions have been discovered that allow extensive deformation of rigid single-walled nanotubes. Nature 2006, 439 (7074), 281281.

[7] Tenne, R.; Margulis, L.; Genut, M.; Hodes, G. Polyhedral and cylindrical structures of tungsten disulfide. Nature 1992, 360 (6403), 444-446.

[8] Rapoport, L.; Fleischer, N.; Tenne, R. Applications of $\mathrm{WS}_{2}\left(\mathrm{MoS}_{2}\right)$ inorganic nanotubes and fullerene-like nanoparticles for solid lubrication and for structural nanocomposites. J. Mater. Chem. 2005, 15 (18), 17821788.

[9] Kaplan-Ashiri, I.; Cohen, S. R.; Gartsman, K.; Rosentsveig, R.; Seifert, G.; Tenne, R. Mechanical behavior of individual WS $_{2}$ nanotubes. J. Mater. Res. 2004, 19 (2), 454-459.

[10] Kaplan-Ashiri, I.; Cohen, S. R.; Gartsman, K.; Ivanovskaya, V.; Heine, T.; Seifert, G.; Wiesel, I.; Wagner, H. D.; Tenne, $\mathrm{R}$. On the mechanical behavior of $\mathrm{WS}_{2}$ nanotubes under axial tension and compression. Proc. Natl. Acad. Sci. U.S.A. 2006, 103 (3), 523-528.

[11] Kaplan-Ashiri, I.; Cohen, S. R.; Apter, N.; Wang, Y. K.; Seifert, G.; Wagner, H. D.; Tenne, R. Microscopic investigation of shear in multiwalled nanotube deformation. J. Phys. Chem. C 2007, 111 (24), 84328436.

[12] Zhu, Y. Q.; Sekine, T.; Li, Y. H.; Fay, M. W.; Zhao, Y.
M.; Poa, C. H. P.; Wang, W. X.; Roe, M. J.; Brown, P. D.; Fleischer, N.; Tenne, R. Shock-absorbing and failure mechanisms of $\mathrm{WS}_{2}$ and $\mathrm{MoS}_{2}$ nanoparticles with fullerene-like structures under shock wave pressure. J. Am. Chem. Soc. 2005, 127 (46), 16263-16272.

[13] Wang, M. S.; Peng, L. M.; Wang, J. Y.; Chen, Q. Shaping carbon nanotubes and the effects on their electrical and mechanical properties. Adv. Funct. Mater. 2006, 16 (11), 1462-1468.

[14] Wang, M. S.; Wang, J. Y.; Chen, Q.; Peng, L. M. Fabrication and electrical and mechanical properties of carbon nanotube interconnections. Adv. Funct. Mater. 2005, 15 (11), 1825-1831.

[15] Gao, R. P.; Wang, Z. L.; Bai, Z. G.; de Heer, W. A.; Dai, L. M.; Gao, M. Nanomechanics of individual carbon nanotubes from pyrolytically grown arrays. Phys. Rev. Lett. 2000, 85 (3), 622-625.

[16] Poncharal, P.; Wang, Z. L.; Ugarte, D.; de Heer, W. A. Electrostatic deflections and electromechanical resonances of carbon nanotubes. Science 1999, 283 (5407), 1513-1516.

[17] Yu, M. F.; Wagner, G. J.; Ruoff, R. S.; Dyer, M. J. Realization of parametric resonances in a nanowire mechanical system with nanomanipulation inside a scanning electron microscope. Phys. Rev. B 2002, 66 (7), 073406.

[18] Gaillard, J.; Skove, M.; Rao, A. M. Mechanical properties of chemical vapor deposition-grown multiwalled carbon nanotubes. Appl. Phys. Lett. 2005, 86 (23), 233109.

[19] Liu, K. H.; Wang, W. L.; Xu, Z.; Liao, L.; Bai, X. D.; Wang, E. G. In situ probing mechanical properties of individual tungsten oxide nanowires directly grown on tungsten tips inside transmission electron microscope. Appl. Phys. Lett. 2006, 89 (22), 221908

[20] Gere, J. M. Mechanics of Materials, 6th ed.; Brooks/Cole Publishing: Pacifie Grove, 2004; p. 940.

[21] Yakobson, B. I.; Avouris, P. Mechanical properties of carbon nanotubes. Carbon Nanotubes-Topics in Applied Physics 2001, 80, 287-327.

[22] Arroyo, M.; Belytschko, T. Nonlinear mechanical response and rippling of thick multiwalled carbon nanotubes. Phys. Rev. Lett. 2003, 91 (21), 215505.

[23] Falvo, M. R.; Clary, G. J.; Taylor, R. M.; Chi, V.; Brooks, F. P.; Washburn, S.; Superfine, R. Bending and buckling of carbon nanotubes under large strain. Nature 1997, 389 (6651), 582-584. 
[24] lijima, S.; Brabec, C.; Maiti, A.; Bernholc, J. Structural flexibility of carbon nanotubes. J. Chem. Phys. 1996, 104 (5), 2089-2092.

[25] Liu, J. Z.; Zheng, Q. S.; Jiang, Q. Effect of bending instabilities on the measurements of mechanical properties of multiwalled carbon nanotubes. Phys. Rev. B 2003, 67 (7), 075414.

[26] Golberg, D.; Costa, P. M. F. J.; Lourie, O.; Mitome, M.; Bai, X. D.; Kurashima, K.; Zhi, C. Y.; Tang, C. C.; Bando, $Y$. Direct force measurements and kinking under elastic deformation of individual multiwalled boron nitride nanotubes. Nano Lett. 2007, 7 (7), 2146-2151.

[27] Kuzumaki, T.; Kitakata, S.; Enomoto, K.; Yasuhara, T.; Ohtake, N.; Mitsuda, Y. Dynamic observation of the bending behavior of carbon nanotubes by nanoprobe manipulation in TEM. Carbon 2004, 42 (11), 2343-2345.

[28] Ding, W.; Calabri, L.; Kohlhaas, K. M.; Chen, X.; Dikin, D. A.; Ruoff, R. S. Modulus, fracture strength, and brittle vs. plastic response of the outer shell of arc-grown multiwalled carbon nanotubes. Exp. Mech. 2007, 47 (1), 2536.

[29] Kuzumaki, T.; Hayashi, T.; Ichinose, H.; Miyazawa, K.; Ito, K.; Ishida, Y. In situ observed deformation of carbon nanotubes. Philos. Mag. 1998, 77 (6), 1461-1469.
[30] Lide, D. R. CRC Handbook of Chemistry and Physics; CRC Press: Cleveland, Ohio, 1977.

[31] Joly-Pottuz, L.; Martin, J. M.; Dassenoy, F.; Belin, M.; Montagnac, G.; Reynard, B.; Fleischer, N. Pressureinduced exfoliation of inorganic fullerene-like $\mathrm{WS}_{2}$ particles in a Hertzian contact. J. Appl. Phys. 2006, 99 (2), 023524/1-023524/5.

[32] Yu, M. F.; Files, B. S.; Arepalli, S.; Ruoff, R. S. Tensile loading of ropes of single wall carbon nanotubes and their mechanical properties. Phys. Rev. Lett. 2000, 84 (24), 5552-5555.

[33] Yu, M. F.; Lourie, O.; Dyer, M. J.; Moloni, K.; Kelly, T. F.; Ruoff, R. S. Strength and breaking mechanism of multiwalled carbon nanotubes under tensile load. Science 2000, 287 (5453), 637-640.

[34] Yu, M. F.; Yakobson, B. I.; Ruoff, R. S. Controlled sliding and pullout of nested shells in individual multiwalled carbon nanotubes. J. Phys. Chem. B 2000, 104 (37), 8764-8767.

[35] Gibson, R. F.; Ayorinde, E. O.; Wen, Y. F. Vibrations of carbon nanotubes and their composites: A review. Compos. Sci. Technol. 2007, 67 (1), 1-28.

[36] Kaplan-Ashiri, I.; Tenne, R. Mechanical Properties of $W_{2}$ Nanotubes J. Clust. Sci. 2007, 18 (3), 549-563. 